

\title{
Quantifying the absorption and reduced scattering coefficients of tissuelike turbid media over a broad spectral range with noncontact Fourier-transform hyperspectral imaging
}

\author{
Tuan H. Pham, Frederic Bevilacqua, Thorsten Spott, Jan S. Dam, Bruce J. Tromberg, \\ and Stefan Andersson-Engels
}

\begin{abstract}
Absorption $\left(\mu_{a}\right)$ and reduced scattering $\left(\mu_{s}^{\prime}\right)$ spectra of turbid media were quantified with a noncontact imaging approach based on a Fourier-transform interferometric imaging system (FTIIS). The FTIIS was used to collect hyperspectral images of the steady-state diffuse reflectance from turbid media. Spatially resolved reflectance data from Monte Carlo simulations were fitted to the recorded hyperspectral images to quantify $\mu_{a}$ and $\mu_{s}^{\prime}$ spectra in the 550-850-nm region. A simple and effective calibration approach was introduced to account for the instrument response. With reflectance data that were close to and far from the source $(0.5-6.5 \mathrm{~mm}), \mu_{a}$ and $\mu_{s}^{\prime}$ of homogeneous, semi-infinite turbid phantoms with optical property ranges comparable with those of tissues were determined with an accuracy of $\pm 7 \%$ and $\pm 3 \%$, respectively. Prediction accuracy for $\mu_{a}$ and $\mu_{s}^{\prime}$ degraded to $\pm 12 \%$ and $\pm 4 \%$, respectively, when only reflectance data close to the source $(0.5-2.5 \mathrm{~mm})$ were used. Results indicate that reflectance data close to and far from the source are necessary for optimal quantification of $\mu_{a}$ and $\mu_{s}^{\prime}$. The spectral properties of $\mu_{a}$ and $\mu_{s}^{\prime}$ values were used to determine the concentrations of absorbers and scatterers, respectively. Absorber and scatterer concentrations of two-chromophore turbid media were determined with an accuracy of $\pm 5 \%$ and $\pm 3 \%$, respectively. (c) 2000 Optical Society of America
\end{abstract}

OCIS codes: $170.0110,170.7050,170.6510,070.2590,120.3180$.

\section{Introduction}

The absorption and reduced scattering parameters ( $\mu_{a}$ and $\mu_{s}^{\prime}$, respectively) of turbid media, such as tissue, can be used to characterize its composition and structure. For instance, $\mu_{a}$ and $\mu_{s}^{\prime}$ of tissue can provide information on a variety of physiological processes. Wavelength-dependent absorption is used to quantify the concentration of biologically important

T. H. Pham, J. S. Dam, and S. Andersson-Engles (Stefan. Andersson-Engels@fysik.lth.se) are with the Department of Physics, Lund Institute of Technology, P.O. Box 118, SE22100 Lund, Sweden. T. H. Pham, F. Bevilacqua, and B. J. Tromberg are with the Beckman Laser Institute and Medical Clinic, Laser Microbeam and Medical Program, 1002 Health Sciences Road East, Irvine, California 92612. T. Spott is with the Department of Physical Electronics, Norwegian University of Science and Technology, N-7304 Trondheim, Norway. J. S. Dam is with Bang \& Olufsen Medicom a/s, DK-7600 Struer, Denmark.

Received 22 February 2000; revised manuscript received 7 September 2000.

0003-6935/00/346487-11\$15.00/0

(C) 2000 Optical Society of America chromophores, such as hemoglobin, myoglobin, water, fat, and near-infrared-absorbing drugs. ${ }^{1-5}$ Wavelength-dependent scattering properties offer insight into the composition, density, and organization of tissue structures, such as cells, subcellular organelles, and connective tissue-extracellular matrices. ${ }^{6-10}$ Since changes in these components generally accompany pathologic transformations and physiologic processes, techniques for noninvasively quantifying $\mu_{a}$ and $\mu_{s}^{\prime}$ in vivo have generated intense interest.

One approach for characterizing $\mu_{a}$ and $\mu_{s}^{\prime}$ of turbid media is to use spatially resolved, steady-state diffuse reflectance. Two strategies for spatially resolved reflectance measurements of turbid, semiinfinite media have been demonstrated: contact probe detection using fiber-optic arrays ${ }^{11-15}$ and image reflectometry. ${ }^{16-19}$ Systems based on contact probe detection often have the capacity to measure the reflectance for a continuous range of wavelengths but require that the detection fibers touch the tissue. Imaging reflectometry has the advantage that measurements can be performed remotely. Noncontact 
detection can be particularly useful in clinical settings where fiber-optic probes may contribute to a number of problems, including contaminating sterile fields, altering regional tissue perfusion (through probe-induced pressure), and increasing measurement uncertainty that is due to irreproducible fibertissue coupling.

When a light source is launched onto tissue, diffuse reflectance signals close to and far from the injection point do not have equal sensitivity to bulk media optical properties. The reflectance profile close to the source, i.e., approximately less than 1 transport length $l_{\mathrm{tr}}=\left(\mu_{a}+\mu_{s}^{\prime}\right)^{-1}$, depends strongly on the phase function, anisotropy factor, scattering property, ${ }^{20,21}$ and source volumetric distribution but exhibits weak dependence on absorption. 15,21,22 Far from the source $\left(>5 l_{\text {tr }}\right)$, diffuse reflectance profiles depend significantly on absorption but less so on the phase function and source volumetric distribution. ${ }^{22,23}$ In addition, photons that are diffusely reflected close to the source mainly probe superficial regions of the medium, whereas photons reflected distal to the source have diffused into deeper regions. ${ }^{24,25}$ Accordingly, parameters such as scattering and anisotropy of superficial regions may be optimally characterized with diffuse reflectance information close to the source. Diffuse reflectance profiles far from the source may be optimal for quantifying the absorption and scattering properties of deeper regions. Thus source-detector separation, i.e., the distance between the source and the location of light reemission, is an important parameter to consider in analyzing diffuse reflectance images. ${ }^{26}$

Imaging reflectometry systems often have limited spectral range, typically consisting of only a few discrete wavelengths. ${ }^{16,17,27}$ For many tissue applications, broad spectral dynamic range is desirable. This is because detailed absorption and scattering spectra can be used to determine the physiological and the structural properties of tissues and are diagnostically valuable in characterizing pathology. ${ }^{28-30}$ Calibration procedures are generally difficult and complex, since the instrument response depends not only on the spatial and the wavelength performance of the CCD but also on the point-spread function of the imaging system. Consequently, techniques that extend the wavelength dynamic range and facilitate rapid, accurate calibration are of considerable interest for spectroscopy and imaging of turbid media.

In this study we investigate the feasibility of using diffusely reflected hyperspectral images to quantify $\mu_{a}$ and $\mu_{s}^{\prime}$ over a continuous and broad spectral range. A Fourier-transform interferometric imaging system (FTIIS) is used to collect hyperspectral diffuse reflectance images from tissuelike phantoms containing Intralipid (a light-scattering fat emulsion) and absorbing dyes. We fit Monte Carlo simulations to measured image data to characterize the wavelength-dependent optical properties of the phantoms. Wavelength-dependent $\mu_{a}$ and $\mu_{s}^{\prime}$ are used to quantify, respectively, dye and fat emulsion concentrations. Additionally, we examine how the range of

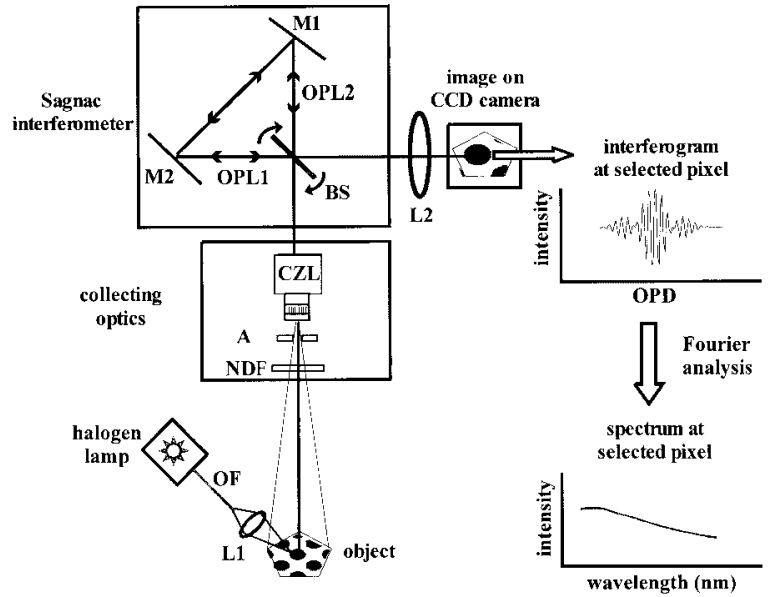

Fig. 1. Experimental setup of the FTIIS used to collect hyperspectral images of the diffuse reflectance from turbid media. The principal components of the system are the halogen white-light source, collection optics, Sagnac interferometer, and a cooled 12-bit CCD camera (CCD). Interference patterns of the object are collected for a sequential series of OPD's by incremental rotation of the beam splitter (BS). An interferogram is generated at each pixel, which corresponds to a particular region of the object. Inverse Fourier transform of the interferograms yields the hyperspectral images. Data acquisition and processing were performed with a personal computer. A full description of the setup is provided in the text. Abbreviations: optical fiber (OF), aperture (A), camera zoom lens (CZL), lens to focus the white-light source (L1), lens to image the recombined beams onto the CCD (L2), mirror $(\mathrm{M})$, and optical path length (OPL).

source-detector separations used in the image analysis affects the determination of $\mu_{a}$ and $\mu_{s}^{\prime}$.

\section{Material and Methods}

\section{A. Fourier-Transform Interferometric Imaging System}

Figure 1 schematically illustrates the main components of the hyperspectral imaging device. White light from a broadband halogen source is coupled into a 0.16-NA, 200- $\mu \mathrm{m}$ diameter optical fiber. Light exiting the fiber is refocused to form a $250-\mu \mathrm{m}$-diameter point source that is launched onto tissuelike phantoms at an incidence angle of 25 deg relative to the surface normal. At this angle, specularly reflected light is not collected by the imaging optics, and the diffuse reflectance distribution is minimally affected. ${ }^{17}$ Hyperspectral images are collected with the FTIIS (SpectraCube imaging system, Applied Spectral Imaging, Migdal HaEmek Model 10511, Israel).

The FTIIS employs a cyclic (Sagnac) interferometer $^{31}$ to construct hyperspectral images. The principal components of the FTIIS are the collection optics, a Sagnac interferometer, and a 12-bit CCD camera (Model TE-CCD-512-EFT/UV, Princeton Instruments, Inc., Trenton, New Jersey). The collection optics consist of a commercial camera zoom lens (50-mm focal length, f1.2-f16) and a neutral-density filter (NDF). The achromatic zoom lens allows for adjustable image magnification and acceptance angle. NDF's were placed in front of the camera lens to 
prevent saturation of the CCD pixels that image the regions near the source, where the intensity is maximal.

Light entering the collection optics is split into two beams and directed along different optical paths (OPL1 and OPL2). The two beams are then recombined at the CCD. The optical path difference (OPD) between the beams, which is a function of the beam splitter's angular position, generates interference patterns of the object on the CCD. To obtain hyperspectral images, interference patterns are collected for sequences of OPD's, generated by stepwise rotation of the beam splitter. In this manner an interferogram is constructed for each pixel in the image. Fourier analysis of the interferograms is used to produce wavelength spectra. The selected magnitude, range, and spacing of the OPD's determine spectral range and resolution. Images can occupy as many as $512 \times 512$ pixels (for a given wavelength), and, depending on the selected magnification, objects with sizes of the order of 1-50 mm can be imaged. In this study we set the FTIIS parameters such that hyperspectral images for wavelengths ranging from 550-850 $\mathrm{nm}$ were generated. Twenty-four wavelengths were obtained with images unevenly spaced within the wavelength range. On average, the spacing was $8 \mathrm{~nm}$ for shorter wavelengths and $17 \mathrm{~nm}$ for longer wavelengths. Image size was $170 \times 170$ pixels, corresponding to a physical (object) dimension of $25 \times 25 \mathrm{~mm}$. At these settings, hyperspectral images were acquired in $40 \mathrm{~s}$.

\section{B. Phantoms}

Fifteen turbid phantoms with unique and randomly selected pairs of $\mu_{a}$ and $\mu_{s}^{\prime}$ were made from absorbing dyes (Nigrosin and Janus Green B, Sigma, St. Louis, Missouri) and fat emulsion scatterers [Intralipid 200 $\mathrm{mg} / \mathrm{ml}(20 \%)$, Pharmacia \& Upjohn Sverige AB, Stockholm, Sweden). The range of optical properties for the phantoms was comparable with that of tissues $^{32}$; i.e., $\mu_{a}$ and $\mu_{s}^{\prime}$ values were $1 \times 10^{-3}-0.20$ $\mathrm{mm}^{-1}$ and $0.30-2.50 \mathrm{~mm}^{-1}$, respectively. For each phantom, known concentrations of aqueous dye solutions were prepared, followed by the addition of Intralipid scatterers. Conventional absorption spectrophotometry was performed on a small sample (2 $\mathrm{ml}$ ) collected from the aqueous dye mixture, i.e., prior to adding scatterer, for independent measurement of $\mu_{\alpha}$. Expected phantom absorption values from 500 to $900 \mathrm{~nm}$ were calculated with these measured extinction coefficients. These calculated values were then used as gold standards in the evaluation of FTIIS accuracy.

Expected reduced scattering $\left(\mu_{s}^{\prime}\right)$ values were calculated according to van Staveren et al., ${ }^{33}$ who used Mie theory to relate the scattering coefficient and anisotropy factor of Intralipid to the optical wavelength. According to that study, the scattering coefficient $\left(\mu_{s}\right)$ and the anisotropy factor $(g)$ of $10 \%$
Intralipid can be expressed as a function of the optical wavelength, $\lambda$ :

$$
\begin{aligned}
& \mu_{s}(\lambda)=16 \lambda^{-2.4}, \\
& g(\lambda)=1.1-0.58 \lambda,
\end{aligned}
$$

where $\lambda$ is given in micrometers and $\mu_{s}$ in inverse millimeters. Values obtained from Eqs. (1) and (2) were shown to have an accuracy of $\pm 6 \%$. The reduced scattering coefficient for $10 \%$ Intralipid was calculated from $\mu_{s}$ and $g$ with the similarity relation, $\mu_{s}^{\prime}=\mu_{s}(1-g)$, which is satisfied when the sourcedetector separation, $\rho$, is greater than approximately one transport length. ${ }^{34} \mu_{s}^{\prime}$ for Intralipid can be expressed as $\mu_{s}^{\prime}=\sigma C_{\%}$, where $\sigma=\mu_{s}(1-g) / 10$ and $C_{\%}$ is the Intralipid concentration in percent volume. This relationship has been shown to be valid when Intralipid concentration is less than $10 \%$ by volume. ${ }^{33}$ Optical property values obtained from fits of the hyperspectral data were compared with expected coefficients to determine the percent accuracy and spectral fidelity of quantifying $\mu_{a}$ and $\mu_{s}^{\prime}$.

\section{Monte Carlo Simulations}

Monte Carlo simulations were performed to generate a database of diffuse reflectance from a finitediameter $(250-\mu \mathrm{m})$ source in homogeneous, semiinfinite media. We elected to use Monte Carlo simulation instead of the diffusion approximation, because the numerical approach readily accounts for the effects of boundary, source distribution, and media geometry on the diffuse reflectance. In addition, reflectance data from Monte Carlo simulations are more accurate for highly absorbing media and for positions close to the source. The HenyeyGreenstein phase function was used in the simulations, since various investigators have used this for successful modeling of the scattering phase function of fat emulsions. The range of optical properties used to generate the database encompassed values typically found in tissues: $\mu_{a}$ and $\mu_{s}^{\prime}$ were $1 \times 10^{-3}$ $0.30 \mathrm{~mm}^{-1}$ and $0.30-2.50 \mathrm{~mm}^{-1}$, respectively, with $\mu_{a}$ equally spaced by $1 \times 10^{-2} \mathrm{~mm}^{-1}$ and $\mu_{s}^{\prime}$ by 0.20 $\mathrm{mm}^{-1}$. The refractive index was set to that of water. Because of cylindrical symmetry, Monte Carlo data for diffuse reflectance and other parameters were organized with cylindrical coordinates. For example, diffuse reflectance data were arranged in concentric rings surrounding the source. The radial bin thickness of the concentric rings was $50 \mu \mathrm{m}$. The number of photons was empirically set at $N=\left(\mu_{a} / \mu_{s}^{\prime}\right)^{1 / 4} \times$ $10^{7}$ so that the noise characteristics of data simulated with different optical properties would be comparable. ${ }^{17}$ Simulated data were normalized to ring area to account for differences in ring size. The diffuse reflectance was recorded as a function of the radial distance from the source and stored as photon probability per unit area.

An interpolation procedure was used to obtain simulated reflectance data for optical properties that were not the same as the simulated values, e.g., for 
values lying in between the simulated $\mu_{a}, \mu_{s}^{\prime}$ pairs. In particular, we used a three-dimensional spline function $^{35,36}$ to model the Monte Carlo reflectance database as a function of $\mu_{a}, \mu_{s}^{\prime}$, and $\rho$. The spline function was then used to interpolate radially resolved reflectance data for arbitrary $\mu_{a}$ and $\mu_{s}^{\prime}$ values within the simulated range.

\section{Data Fitting}

Monte Carlo simulations were fitted to the hyperspectral images to determine $\mu_{a}$ and $\mu_{s}^{\prime}$ values in the visible and the near-infrared regions. Prior to data fitting, a calibration procedure was performed to account for the instrument response (IR) of the imaging system, i.e., instrument factors such as the pointspread function, spectral sensitivity of the CCD, and uniformity of the CCD response. The calibration procedure consisted of three main steps. First, we collected an image of a marked ruler and counted the number of pixels corresponding to a unit length on the ruler. The ratio of length to pixel count is the factor that relates the pixel count to the physical size of the object. Second, hyperspectral images of the diffuse reflectance were acquired from a reference phantom, which was constructed from Intralipid and dyes, to determine the IR's at the measured wavelengths. Calibration images were obtained with experimental settings, such as numerical aperture and magnification factor, that were identical to those used to construct hyperspectral images of the test samples. Third, the IR of the system was calculated with the calibration images and the known optical properties of the reference phantom. For this purpose the calibration image at each wavelength was Fourier transformed into the spatial-frequency domain (SFD). Likewise, the Monte Carlo simulated image (MCSI) corresponding to the reference phantom optical properties was transformed into the SFD. The overall instrument response of the FTIIS at each wavelength was calculated from the spatial transforms of the calibration and the MCSI with the relation

$$
\mathscr{F}(\mathrm{IR})=\mathscr{F}(\mathrm{I}) / \mathscr{F}(\mathrm{MCSI}),
$$

where $\mathscr{F}(\mathrm{I}), \mathscr{F}(\mathrm{MCSI})$, and $\mathscr{F}(\mathrm{IR})$ are the spatial Fourier transforms of the calibration image, the MCSI, and the IR, respectively.

To perform data fitting, the MCSI corresponding to a particular set of optical properties was effectively convolved with the IR at the evaluated wavelength, i.e., MCSI $\otimes I R$ where the symbol $\otimes$ denotes a convolution. However, the convolution was performed indirectly in the SFD. The MCSI was first transformed into the SFD, F्F(MCSI). The spatialfrequency transform of the MCSI was multiplied by $\mathscr{F}(\mathrm{IR})$ to account for the instrument response. The product, $\mathscr{F}(\mathrm{MCSI}) \mathscr{F}(\mathrm{IR})$, was inversely Fourier transformed into the spatial domain to yield MCSI $\otimes$ IR. The sample image at each wavelength and MCSI $\otimes I R$ were binned radially. That is, the images were divided into concentric rings, and pixel values in each ring were summed and normalized to the pixel count of the ring. Radial binning of data conferred two advantages: (i) pixel values from different quadrants were averaged and thus noise was reduced, and (ii) computational demand of fitting was reduced, since fitting was performed on data of one dimension instead of two. A simplex minimization algorithm (Nelder-Meads) was used to perform least-squares fitting of the radially binned MCSI $\otimes$ IR to the corresponding binned sample image. Fitting of the hyperspectral data was performed independently on one image at a time and proceeded sequentially until all images of the spectrum were fitted. Different initial guess values of the fit parameters $\left(\mu_{a}\right.$ and $\left.\mu_{s}^{\prime}\right)$ were used to avoid local minima, and the fit parameters were selected from the trial with the lowest least-squares residuals.

To examine how the range of source-detector separations affects the accuracy of quantifying $\mu_{a}$ and $\mu_{s}^{\prime}$, we fitted the MCSI to sample data for various ranges of $\rho$. Three ranges of $\rho$ were examined: (i) distances covering the full range $(0.5-6.5 \mathrm{~mm})$, (ii) distances close to the source $(0.5-2.5 \mathrm{~mm})$, and (iii) distances far from the source $(4-6.5 \mathrm{~mm})$.

\section{E. Concentrations}

The $\mu_{a}$ spectra obtained from the fit were used to determine the dye concentrations. $\mu_{a}$ values are related to the dye concentrations as expressed by the matrix

$$
\left(\begin{array}{c}
\mu_{a}^{\lambda 1} \\
\mu_{a}^{\lambda 2} \\
\cdot \\
\cdot \\
\mu_{a}^{\lambda n}
\end{array}\right)=\left[\begin{array}{ccccc}
\epsilon_{d 1}^{\lambda 1} & \epsilon_{d 2}^{\lambda 1} & \cdot & \cdot & \epsilon_{d m}^{\lambda 1} \\
\epsilon_{d 1}^{\lambda 2} & \cdot & & & \cdot \\
\cdot & \cdot & & \cdot \\
\cdot & \cdot & & & \cdot \\
\epsilon_{d 1}^{\lambda n} & \epsilon_{d 2}^{\lambda n} & \cdot & \cdot & \epsilon_{d m}^{\lambda n}
\end{array}\right]\left(\begin{array}{c}
C_{d 1} \\
C_{d 2} \\
\cdot \\
\cdot \\
C_{d m}
\end{array}\right)
$$

where $\epsilon$ is the extinction coefficient $\left(\mathrm{ml} / \mathrm{mg} \mathrm{mm}^{-1}\right)$ of the dye, $d i$, at wavelength $\lambda i$ and $C$ is the dye concentration $(\mathrm{mg} / \mathrm{ml})$. In this study the concentration vector consisted of two elements, since only two dyes were used to make the phantoms. Extinction coefficients of Nigrosin and Janus Green over the wavelength range were determined from absorption spectrophotometer measurements. A least-squares solution of Eq. (4) was determined for the concentration vector, with the constraint that the concentration values be positive. ${ }^{37}$ Within a certain range, the macroscopic $\mu_{s}^{\prime}$ is proportional to the scatterer concentration and can be expressed in matrix form similar to Eq. (4). The fit-derived $\mu_{s}^{\prime}$ spectra were then used to determine percent Intralipid in a manner analogous to the dye concentration calculation.

All programs were written in the Matlab environment, except for the Monte Carlo algorithm, which was written in C, compiled and linked to, and made executable within, the Matlab environment.

\section{Results}

The hyperspectral images of 15 tissuelike phantoms were examined to select an outer distance to be used in the fit. An outer distance of $6.5 \mathrm{~mm}$ was selected 
(a)

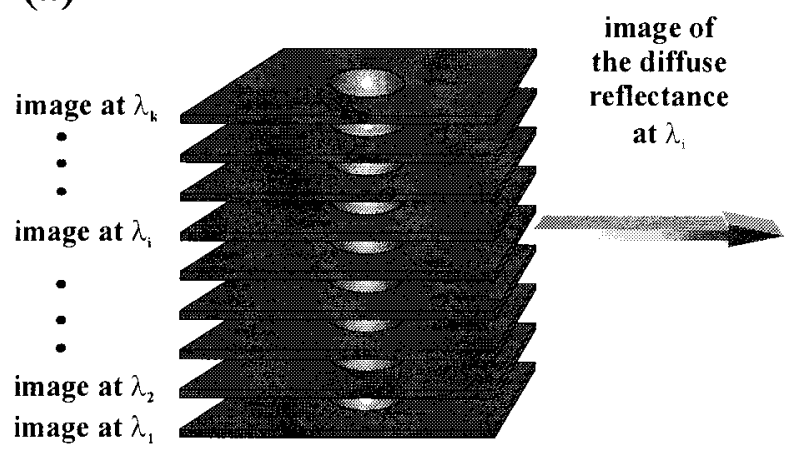

(b)

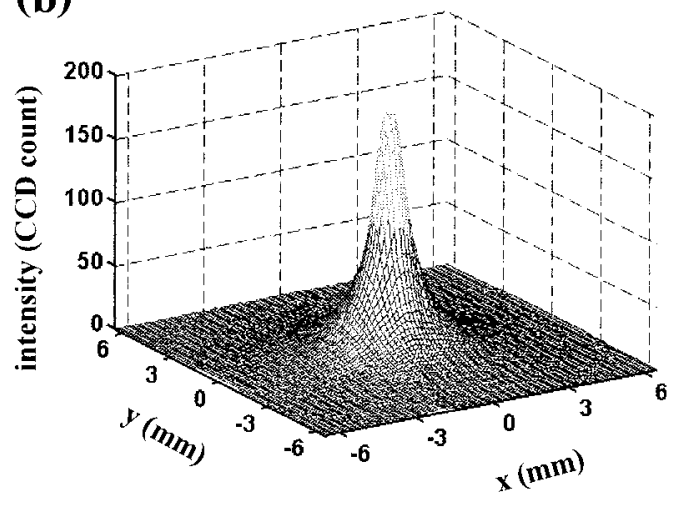

Fig. 2. (a) Schematic showing the data organization of hyperspectral images obtained from FTIIS measurements. Image at each wavelength may contain as many as $512 \times 512$ pixels at 12-bit dynamic range. (b) Typical image of the diffuse reflectance collected on a phantom with $\mu_{a}=0.0281 \mathrm{~mm}^{-1}$ and $\mu_{s}^{\prime}=1.163 \mathrm{~mm}^{-1}$ at $630 \mathrm{~nm}$. NDF's were placed in front of the imaging optics to prevent CCD saturation near the source.

to ensure that the image intensities used in data fitting were higher than the CCD dark noise, and the signal-to-noise ratio was on average greater than 2 at the outer distances $(\sim 6.5 \mathrm{~mm})$. Because of the broad range of spectral data collected from the phantoms, it is not practical to show the full range of data for all the phantoms. Accordingly, the data from the figures are selected from different phantoms at various wavelengths to highlight examples that are representative of the complete data set. Figure 2(a) schematically presents the organization of the hyperspectral image data. In Fig. 2(b) the image intensity is plotted as a function of spatial coordinates $(x, y)$ for a representative diffuse reflectance image. The image was collected from a medium with $\mu_{a}=0.028$ $\mathrm{mm}^{-1}$ and $\mu_{s}^{\prime}=1.16 \mathrm{~mm}^{-1}$ at $630 \mathrm{~nm}$. The uncalibrated image contained contributions from both the diffuse reflectance and the instrument response. An example of the instrument response obtained from calibration measurement is shown in Fig. 3(a) for 630 $\mathrm{nm}$, labeled IR.

Figure 3 depicts the procedure for fitting the MCSI to the sample image. The MCSI's for a set of $\mu_{a}$ and $\mu_{s}^{\prime}$ values were interpolated from the Monte Carlo database. The MCSI's were convolved with the IR to form MCSI $\otimes I R$. Figure 3(a) shows an example of the MCSI and MCSI $\otimes I R$ for $\mu_{a}$ and $\mu_{s}^{\prime}$ values of 0.028 $\mathrm{mm}^{-1}$ and $1.16 \mathrm{~mm}^{-1}$, respectively. As shown in Fig. 3(b), the sample images and MCSI $\otimes I R$ were radially binned, and the binned data were then fitted for the specified distances from the source. Figure 4 shows typical fit results for intensity versus $\rho$ with three ranges of $\rho$ : (a) $0.5-6.5 \mathrm{~mm}$, (b) $0.5-2.5 \mathrm{~mm}$, and (c) $4.0-6.5 \mathrm{~mm}$. In the interest of showing representative data at various wavelengths and optical property values, the data for Fig. 4 are selected from a sample with $\mu_{a}=0.0708 \mathrm{~mm}^{-1}$ and $\mu_{s}^{\prime}=0.316$ $\mathrm{mm}^{-1}$ at $745 \mathrm{~nm}$.

Representative $\mu_{a}$ and $\mu_{s}^{\prime}$ results derived from fitting of reflectance data over the full range of $\rho$ are shown in Fig. 5. Data are acquired from two differ- ent phantoms with $\mu_{a}$ values that span from low to high absorption. Results from the phantom with low $\mu_{a}$ values are shown in Figs. 5(a) and 5(b). Specifically, panel (a) plots the fitted versus expected $\mu_{a}$ values from 550 to $850 \mathrm{~nm}$, and panel (b) plots the fit versus expected values for $\mu_{s}^{\prime}$. Similarly, fit versus expected $\mu_{\alpha}$ values for the highly absorbing phantom are plotted in panel (c), and fit versus expected $\mu_{s}^{\prime}$ values are plotted in panel (d). Diamonds are fit values, dashed curves are expected values, and solid curves are the constrained least-squares solution of Eq. (4) to the fit-derived data to yield concentrations.

Fit values $\mu_{a}$ and $\mu_{s}^{\prime}$ are in excellent agreement with expected values for wavelengths in the range of $550-850 \mathrm{~nm}$. The percent error between the expected and the fit optical property was calculated at each wavelength for all 15 samples. We pooled the percent error values from all samples and calculated the root-mean-square error, which we defined as the percent accuracy in quantifying $\mu_{a}$ and $\mu_{s}^{\prime}$. Table 1 summarizes the percent accuracy for quantifying $\mu_{\alpha}$ and $\mu_{s}^{\prime}$ with three sets of fitting distances, i.e., column 1 for $\rho$ from 0.5 to $6.5 \mathrm{~mm}$, column 2 for $0.5<\rho<2.5$ $\mathrm{mm}$, and column 3 for $4.0<\rho<6.5 \mathrm{~mm}$. The optimal result was obtained using $\rho$ from 0.5 to $6.5 \mathrm{~mm}$, with the percent accuracy for $\mu_{a}$ and $\mu_{s}^{\prime}$ of $\pm 7 \%$ and $\pm 3 \%$, respectively. When reflectance data close to the source $(0.5<\rho<2.5 \mathrm{~mm})$ were fitted, the accuracy for $\mu_{\alpha}$ degraded to $\pm 12 \%$ while $\mu_{s}^{\prime}$ accuracy remained unchanged. The percent accuracies for $\mu_{a}$ and $\mu_{s}^{\prime}$ were significantly worse when the fitting was performed only on the reflectance data distal to the source $(4.0<\rho<6.5 \mathrm{~mm})$.

To examine how sensitive prediction accuracy is to absorption $\left(\mu_{a}\right)$, results from the full-range fit were further divided. Specifically, $\mu_{a}$ and $\mu_{s}^{\prime}$ data from all phantoms at all wavelengths were pooled and grouped into four absorption ranges: $0-0.05 \mathrm{~mm}^{-1}$, $0.05-0.10 \mathrm{~mm}^{-1}, 0.10-0.15 \mathrm{~mm}^{-1}$, and $0.15-0.20$ $\mathrm{mm}^{-1}$. The percent accuracies for $\mu_{a}$ and $\mu_{s}^{\prime}$ within each range were calculated and tabulated in Table 2. 
MCSI

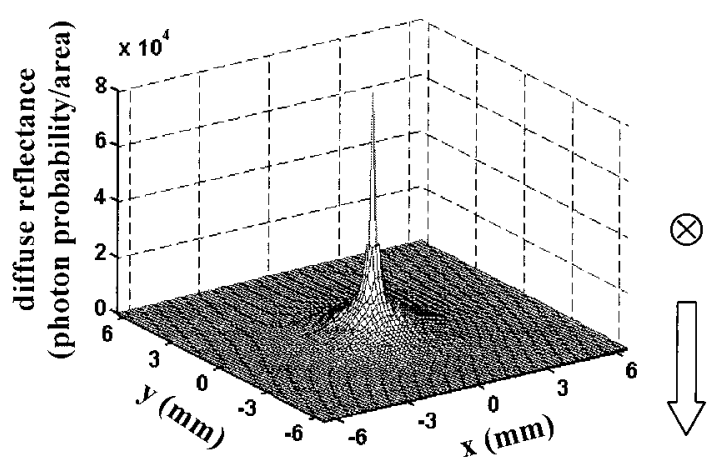

(a)

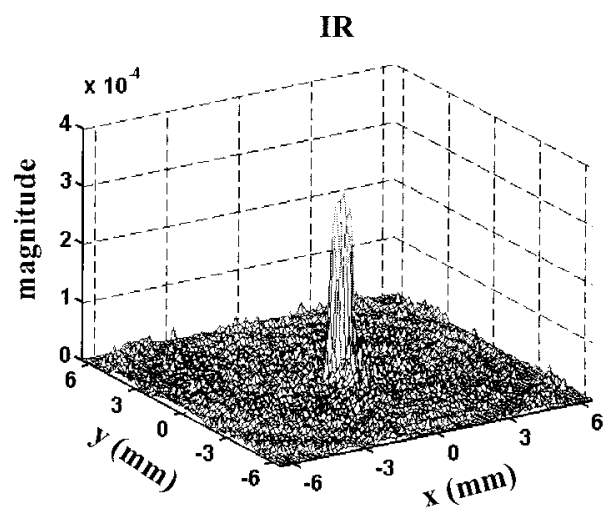

MCSI $\otimes I R$

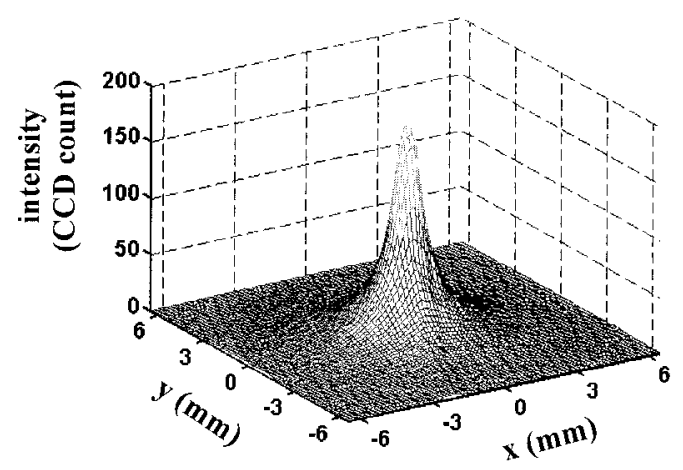

MCSI $\otimes \mathbf{I R}$

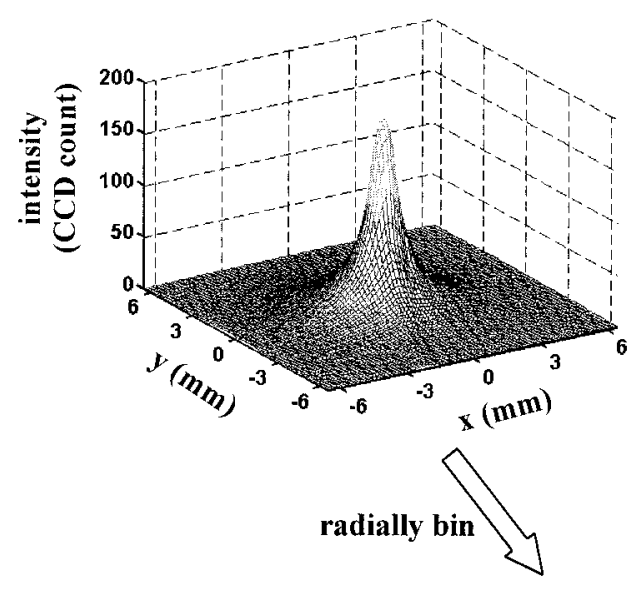

(b)

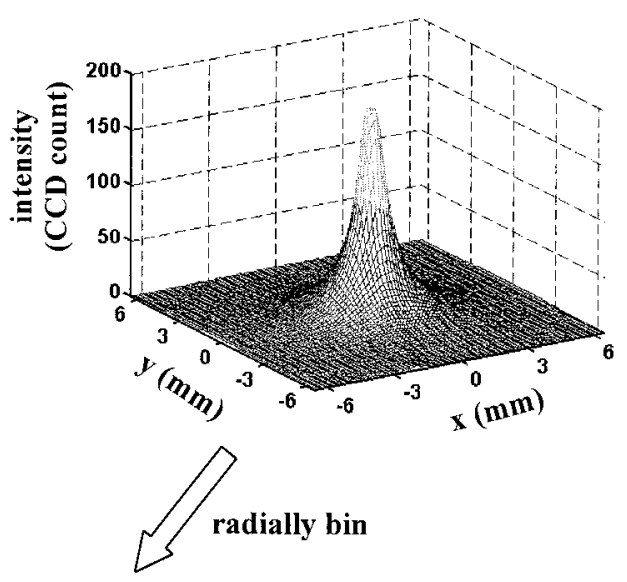

fit radially binned $M C S I \otimes I R$ to binned diffuse reflectance data using simplex minimization algorithm

Fig. 3. (a) Example of (i) the MCSI for $\mu_{a}$ and $\mu_{s}^{\prime}$ values of $0.028 \mathrm{~mm}^{-1}$ and $1.16 \mathrm{~mm}^{-1}$, respectively, and (ii) FTIIS IR's at $630 \mathrm{~nm}$. IR's were calculated from the hyperspectral images collected on a reference phantom and the corresponding MCSI generated from its optical properties. To fit Monte Carlo simulations to the image of the diffuse reflectance, the MCSI was first convolved with IR at the appropriate wavelength to generate MCSI $\otimes I R$. (b) Sample image of the diffuse reflectance and MCSI $\otimes$ IR are radially binned, thereby taking advantage of radial symmetry to reduce computational demands. Radially binned reflectance profiles were then fitted by use of the simplex minimization algorithm.

For the fixed range of distances used, the percent accuracies for media with low absorption were worse than the accuracy values for media with high absorption.

Absorption, $\mu_{\alpha}$, and scattering, $\mu_{s}^{\prime}$, values from the full-range fit were used to calculate, respectively, the phantom dye and Intralipid concentrations. Examples of the constrained least-squares solution to Eq. (4) are shown as solid curves in Fig. 5. For the particular sample shown in Figs. 5(a) and 5(b), the constrained least-squares solution yielded Nigrosin, Janus Green, and Intralipid concentrations of 0.0014 

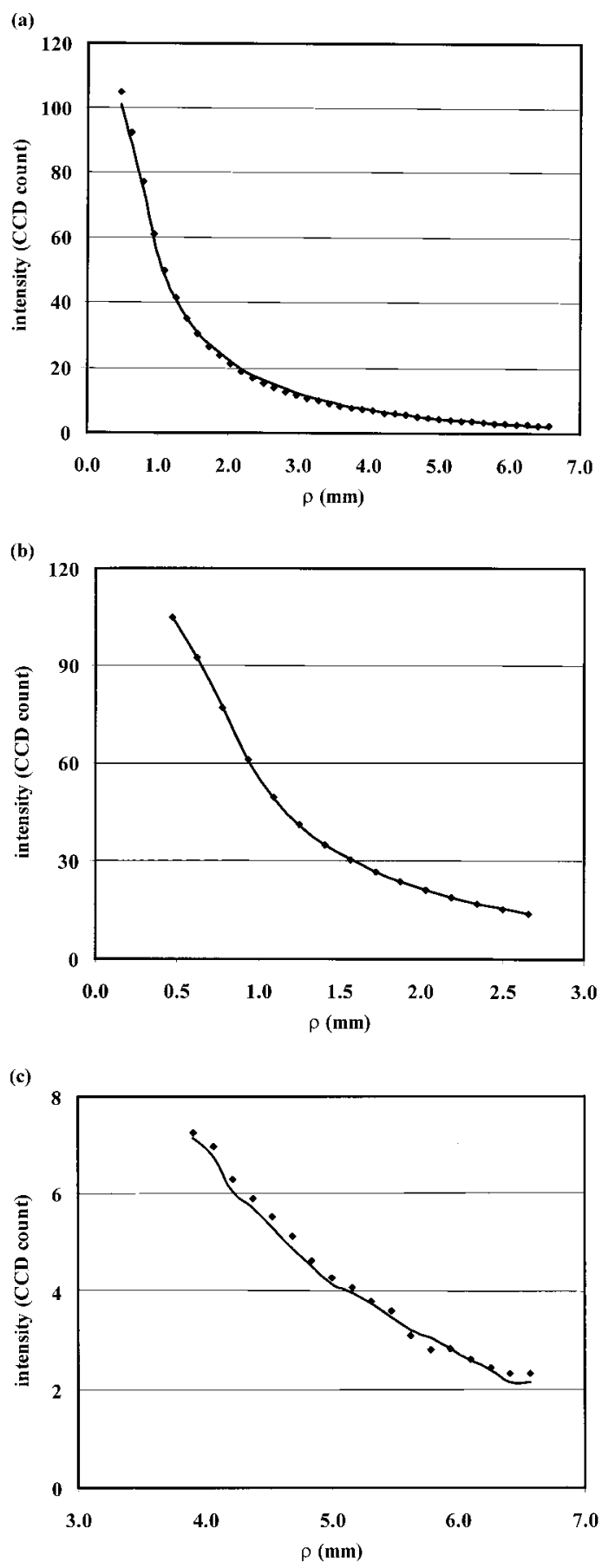

Fig. 4. Examples showing typical results from fitting radially binned MCSI $\otimes$ IR to radially binned diffuse reflectance data at 745 $\mathrm{nm}$ : (a) $\rho$ from 0.5 to $6.5 \mathrm{~mm}$, (b) $\rho$ from 0.5 to $2.5 \mathrm{~mm}$, and (c) $\rho$ from 4.0 to $6.5 \mathrm{~mm}$. Diffuse reflectance data are from a phantom with expected $\mu_{a}$ and $\mu_{s}^{\prime}$ of $0.0708 \mathrm{~mm}^{-1}$ and $0.316 \mathrm{~mm}^{-1}$, respectively, at $745 \mathrm{~nm}$. Note that the binned reflectance and the Monte Carlo simulated data contain significantly more noise at large $\rho$ as compared with the shorter distances.

$\mathrm{mg} / \mathrm{ml}, 0.0324 \mathrm{mg} / \mathrm{ml}$, and $1.01 \%$, respectively. These values deviated from the expected concentrations by approximately $4 \%$ for Nigrosin, $6 \%$ for Janus Green, and 1\% for Intralipid. Measured versus expected concentrations for the 15 phantoms are plot- ted in Fig. 6: (a) Nigrosin, (b) Janus Green, and (c) Intralipid. The measured concentrations for Nigrosin and Janus Green agreed with the expected values to within $\pm 5 \%$, whereas the measured Intralipid concentrations agreed with the expected values to within $\pm 3 \%$.

\section{Discussion and Conclusion}

Quantitative, hyperspectral imaging of diffuse reflectance from turbid media offers several key advantages over contact fiber probe strategies. First, the image contains a complete two-dimensional profile of diffuse reflectance over a continuous and adjustable range of distances from the source. This feature may be important in the spectroscopy of biological tissues, because the full image offers the possibility of identifying inhomogeneous regions. Second, image detection can be performed in noncontact or remote mode, an important feature well suited for certain clinical measurements. Third, conventional bright field images of the medium can be collected in conjunction with diffuse reflectance, thus enabling simultaneous visualization of superficial structure and underlying composition. Finally, the FTIIS has an intrinsically broad spectral dynamic range (visible to near-infrared), overcoming limitations of single or multisource imaging devices. Consequently, the FTIIS is effective for both imaging and spectroscopy of turbid media, such as tissues.

In this study we have demonstrated that $\mu_{a}$ and $\mu_{s}^{\prime}$ of homogeneous turbid media can be accurately determined from FTIIS hyperspectral images of diffuse reflectance. In the process we show that the calibration method used to account for the instrument response is both simple and effective. When Monte Carlo simulations are fitted to hyperspectral images, $\mu_{a}$ and $\mu_{s}^{\prime}$ in the visible and near-infrared can be determined with accuracies of $\pm 7 \%$ and $\pm 3 \%$, respectively. These values are applicable to homogenous, turbid media of semi-infinite geometry with optical properties in the specified range $\left(\mu_{a}\right.$ and $\mu_{s}^{\prime}=1 \times$ $10^{-3}-0.30 \mathrm{~mm}^{-1}$ and $0.30-2.50 \mathrm{~mm}^{-1}$, respectively). Moreover, for a fixed range of source-detector separation, $\mu_{a}$ accuracy degrades when the medium has low absorption. It remains to be determined whether these accuracies can be obtained for $\mu_{a}$ and $\mu_{s}^{\prime}$ in the case of heterogeneous tissues with more complex geometries. Similarly, accuracy values for fits that use small source-detector separations may change when other phase functions are assumed. However, since Monte Carlo solutions to the transport equation were used to model diffuse reflectance, we expect that this technique can be easily extended to media with other geometries, optical property ranges, layered structures, and phase functions.

An important goal of this research was to determine chromophore concentration(s) despite the confounding contribution of multiple light scattering. $\mu_{a}$ and $\mu_{s}^{\prime}$ spectra were used to quantify, respectively, the concentration of absorbers and scatterers in tissue phantoms. Dye concentrations were determined with an accuracy of $\pm 5 \%$, whereas the 
(a)

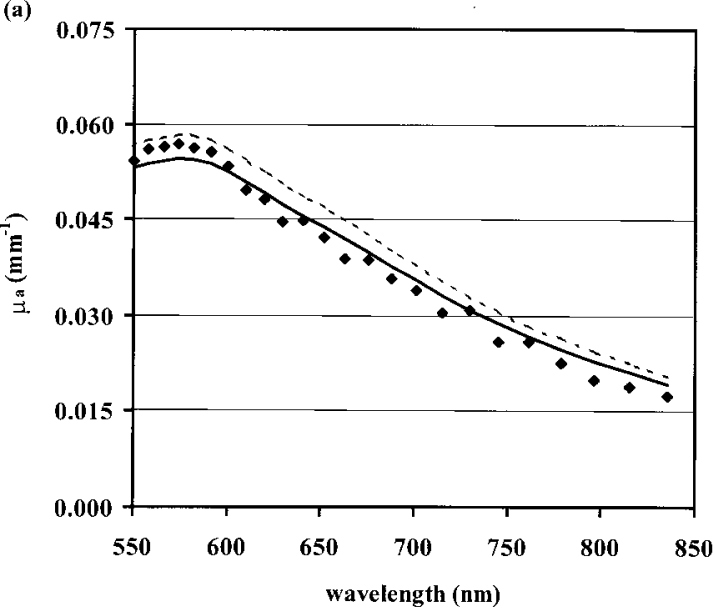

(c)

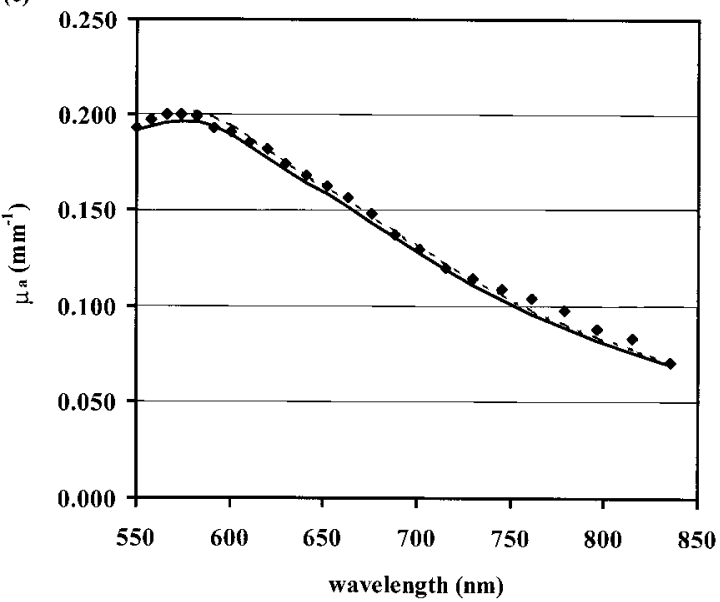

(b)

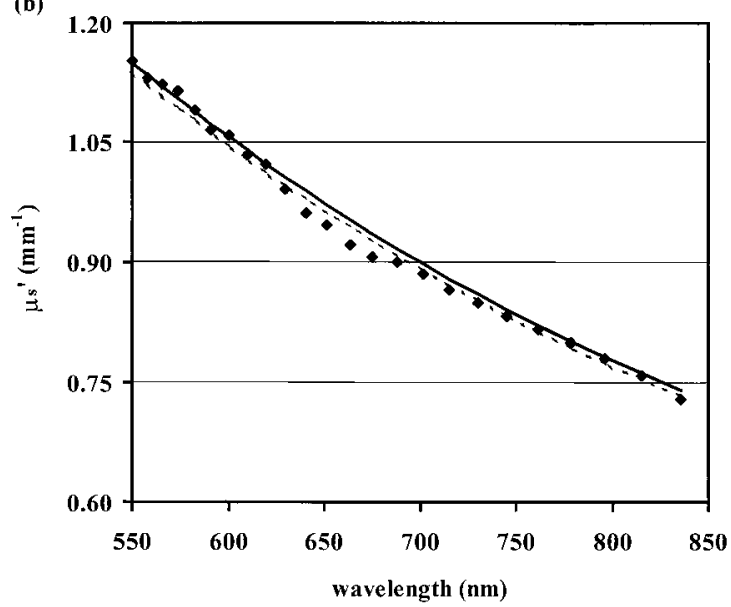

(d)

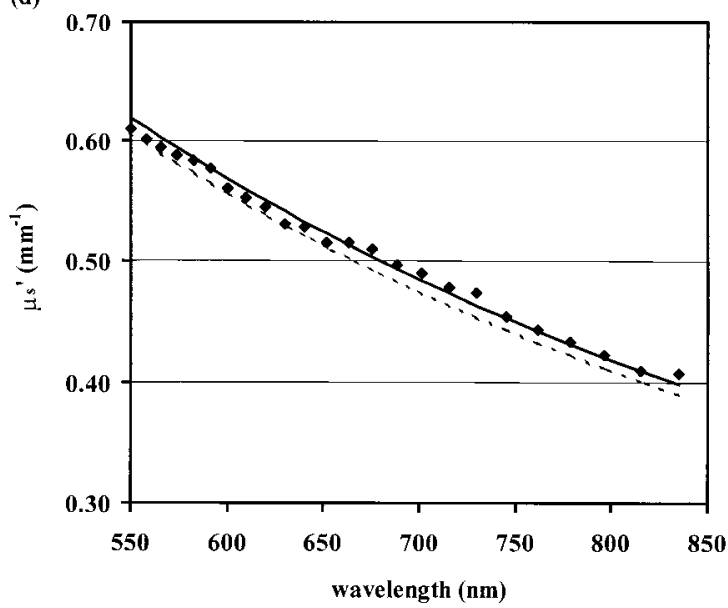

Fig. 5. Fit-derived (diamond) and expected (dashed curves) $\mu_{a}$ and $\mu_{s}^{\prime}$ values from one of the phantoms are plotted as a function of wavelengths. Solid curves, constrained least-square solution to Eq. (4) that was used to determine the concentrations from the spectra of fit values. Data for (a) and (b) are from a representative phantom with low absorption values, whereas data for (c) and (d) are from a phantom with high absorption values. Expected $\mu_{a}$ values were determined from the chromophore concentrations and independently verified with an absorption spectrophotometer. Expected $\mu_{s}^{\prime}$ values were determined from the phantom's Intralipid percent volume. Fit $\mu_{a}$ and $\mu_{s}^{\prime}$ values for the measured wavelengths agree well with expected values. On the basis of the results from 15 phantoms, the constrained least-squares solution to the fit $\mu_{a}$ and $\mu_{s}^{\prime}$ data yielded concentration values for the dyes and Intralipid that were within $5 \%$ and $3 \%$ of the true concentrations, respectively.

Intralipid volume was determined with an accuracy of $\pm 3 \%$.

We also examined the effect of source-detector separation on the accuracy of quantifying $\mu_{a}$ and $\mu_{s}^{\prime}$. Our results suggest that $\mu_{a}$ and $\mu_{s}^{\prime}$ accuracies are optimal when the distances used in the fit encompass spatial regions close to $\left(\leq 0.5 l_{\mathrm{tr}}\right)$ and far $\left(\geq 5 l_{\mathrm{tr}}\right)$ from the source, provided of course, that the reflectance signals remain larger than the noise. This result is consistent with a number of previously reported observations. ${ }^{15,22,23,26,38,39}$ The accuracy of determining $\mu_{a}$ degraded slightly $( \pm 12 \%)$ when we fitted only the diffuse reflectance data close to the source, whereas $\mu_{s}^{\prime}$ accuracy was minimally affected $( \pm 4 \%)$. Interestingly, results from reflectance data close to the source support a previous finding that small

Table 1. Percent Accuracy of Quantifying $\mu_{a}$ and $\mu_{s}^{\prime}$ for Different Range of Fitting Distances

Distances Close to and Far from Source

$0.5 \mathrm{~mm}<\rho<6.5 \mathrm{~mm}$ $\left(\sim 0.25 l_{\text {tr }}\right.$ to $\left.\sim 16 l_{\text {tr }}\right)$
Only Distances Close to Source

$0.5 \mathrm{~mm}<\rho<2.5 \mathrm{~mm}$ $\left(\sim 0.25 l_{\text {tr }}\right.$ to $\left.\sim 5 l_{\text {tr }}\right)$
Only Distances Far from Source

$4.0 \mathrm{~mm}<\rho<6.5 \mathrm{~mm}$ $\left(\sim 8 l_{\text {tr }}\right.$ to $\left.\sim 16 l_{\text {tr }}\right)$

\begin{tabular}{lrrr}
$\pm \mu_{a}(\%)$ & 7 & 12 & 29 \\
$\pm \mu_{s}^{\prime}(\%)$ & 3 & 4 & 23 \\
\hline
\end{tabular}


Table 2. Percent Accuracy of Quantifying $\mu_{a}$ and $\mu_{s}^{\prime}$ for Media with Different Range of Absorption ${ }^{a}$

\begin{tabular}{lcccc}
\hline & \multicolumn{4}{c}{$\mu_{a}$ Range $\left(\mathrm{mm}^{-1}\right)$} \\
\cline { 2 - 5 } & $0.001-0.05$ & $0.05-0.10$ & $0.10-0.15$ & $0.15-0.20$ \\
\hline$\pm \mu_{a}(\%)$ & 11 & 6 & 7 & 3 \\
$\pm \mu_{s}^{\prime}(\%)$ & 5 & 3 & 3 & 4 \\
\hline
\end{tabular}

${ }^{a}$ Results are from the fit that used the full range of distances. The majority of the data is within the $\mu_{a}$ ranges of $0.05-0.10 \mathrm{~mm}^{-1}$ and $0.10-0.15 \mathrm{~mm}^{-1}$.

source-detector separations can yield accurate optical properties. ${ }^{14}$

However, careful consideration of the phase function is essential when small source-detector separations $\left(\approx 1 l_{\text {tr }}\right)$ are used. Indeed, in such a case, the diffuse reflectance is sensitive to the shape of the scattering phase function. ${ }^{20,22,40}$ As shown by Bevilacqua and Depeursinge ${ }^{20}$ for source-detector separations around one transport mean-free path, the important parameter of the phase function to be considered is the ratio $(1-g) /\left(1-g_{2}\right)$, where $g_{2}$ is the second moment of the phase function (the anisotropy factor, $g$, is the first moment of the phase function). The scattering phase function of Intralipid has been successfully modeled by various phase functions matching the first and the second moment of the Henyey-Greenstein phase function. ${ }^{41}$ Moreover, the excellent accuracies obtained in this study support the validity of the Henyey-Greenstein phase function for such fat emulsions. Accuracy values for determining $\mu_{a}$ and $\mu_{s}^{\prime}$ quoted in this study may worsen if an inaccurate phase function is used, ${ }^{14,20}$ particularly for fits that use only the short sourcedetector separations. Nevertheless, it must be noted that using the same type of media for the calibration and the measurements may also significantly reduce errors, since, under these conditions, phase function differences should not exist.

Far from the source, the effects of noise play a critical role in the accurate quantification of $\mu_{a}$ and $\mu_{s}^{\prime}$ for several reasons. First, the decay of the reflectance profile is approximately given by $\exp \left(-\rho / l_{\text {tr }}\right)$, making it difficult to separate $\mu_{a}$ from $\mu_{s}^{\prime}$ because of the single decay constant. Second, the signal-tonoise ratio decreases dramatically with $\rho$, because the diffuse reflectance intensity decays, but the CCD noise remains relatively constant. Finally, the signal-to-noise in the Monte Carlo simulation decreases for larger distances, since fewer photons are collected at distances far from the source. Note that the latter two reasons are practical and not fundamental limitations. The signal-to-noise ratio of the diffuse reflectance data and the Monte Carlo simulations can be improved on, respectively, by use of a low-noise, high-dynamic-range CCD and launching of more photons during the simulation step. In practical terms, however, these factors contribute to poor accuracy only when fits are performed on reflectance data far from the source. Consequently, optimal fit
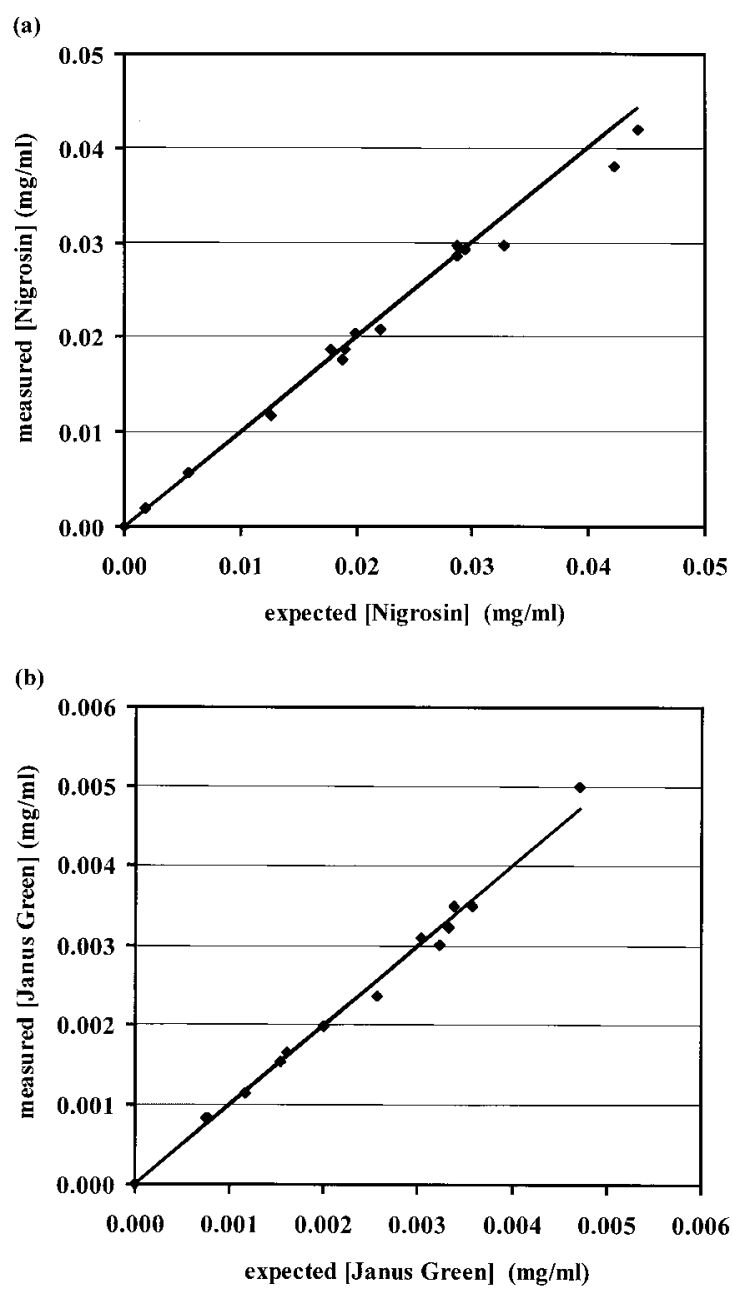

(c)

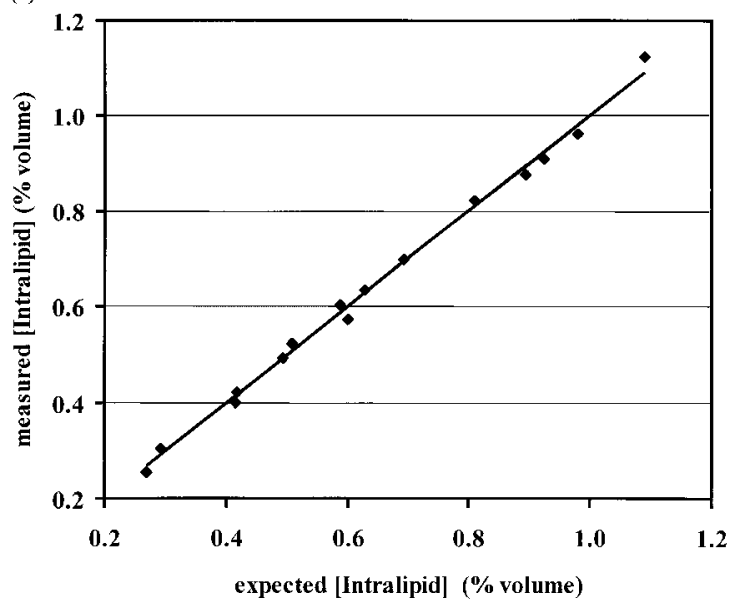

Fig. 6. Least-squares method was used to fit the absorption values in the range of $550-850 \mathrm{~nm}$ to determine the chromophore concentrations of the phantoms. Similarly, Intralipid percent volume was determined from wavelengthdependent fit $\mu_{s}^{\prime}$ values. Measured (diamond) and expected (solid curves) values for (a) Nigrosin concentration, (b) Janus Green concentration, and (c) Intralipid percent volume are plotted for the 15 phantoms. Measured concentrations agree well with expected values. Differences between measured and expected concentrations yield the percent accuracy values of $\pm 5 \%$, $\pm 5 \%$, and $\pm 3 \%$ for Nigrosin, Janus Green, and Intralipid, respectively. 
results are obtained when reflectance data both close to and far from the source as used. This conclusion is reasonable when we take into consideration the instrumental limitations as well as complications that arise from an incomplete knowledge of the phase function for a particular medium.

For reliable recording of data from distances close to and far from the source, a high-dynamic-range $\mathrm{CCD}$ is required. Since these systems are quite expensive, an alternative approach could involve use of a specially designed neutral-density filter (NDF) that has variable attenuation along the radial direction; i.e., the optical density is high near the center and decreases radially away from the center. This would compensate for the rapid attenuation of the diffuse reflectance away from the source, create a flatter image intensity decay profile, and reduce the need for using a high-dynamic-range CCD.

At present, direct least-squares fitting of the Monte Carlo simulations to the data is too computationally intensive for rapid analysis of the hyperspectral images. Data fitting of the hyperspectral images with a simplex algorithm requires as much as $0.5 \mathrm{~h}$ on a $400-\mathrm{MHz}$ Pentium II personal computer. Note that the computational demands are primarily due to the least-squares fits, not the Monte Carlo simulations. In fact, the interpolation algorithm used to generate the simulated data from the Monte Carlo database is computationally efficient and rapid. Alternative approaches to direct fitting may significantly decrease computational demands. One such empirical approach could involve analyzing hyperspectral reflectance images with chemometrics, e.g., partial least squares. In the future we plan to examine the feasibility of this strategy for directly calculating optical properties and chromophore concentrations. With continued improvements in modulating CCD dynamic range requirements and enhanced data analysis we expect that the FTIIS will find important applications in noncontact tissue spectroscopy.

The authors thank Jan Åke Jönsson for providing access to the spectrophotometer and Philippe Thueler for the insightful discussions on data fitting. This study was supported by the Swedish Research Council for Engineering Sciences, the Knut and Alice Wallenberg Foundation, and the National Institutes of Health Laser Microbeam and Medical Program (NIH RR01192). T. H. Pham acknowledges support provided by the Fulbright Fellowship and the Whitaker Foundation Graduate Fellowship. F. Bevilacqua acknowledges support from the Swiss National Science Foundation.

\section{References}

1. B. J. Tromberg, R. C. Haskell, S. J. Madsen, and L. O. Svaasand, "Characterization of tissue optical properties using photon density waves," Comments Mol. Cell. Biophys. 8, 359386 (1995).

2. C. E. Elwell, M. Cope, A. D. Edwards, J. S. Wyatt, D. T. Delpy, and E. O. R. Reynolds, "Quantification of adult cerebral hemodynamics by near-infrared spectroscopy,” J. Appl. Physiol. 77, 2753-2760 (1994).
3. B. Chance, "Near-infrared images using continuous, phasemodulated, and pulsed light with quantitation of blood and blood oxygenation," Ann. N. Y. Acad. Sci. 838, 29-45 (1998).

4. J. Fishkin, P. T. C. So, A. E. Cerussi, S. Fantini, M. A. Franceschini-Fantini, and E. Gratton, "Frequency-domain method for measuring spectral properties in multiplescattering media: methemoglobin absorption spectrum in a tissuelike phantom,” Appl. Opt. 34, 1143-1155 (1995).

5. R. A. Weersink, J. E. Hayward, K. R. Diamond, and M. S. Patterson, "Accuracy of noninvasive in vivo measurements of photosensitizer uptake based on a diffusion model of reflectance spectroscopy," Photochem. Photobiol. 66, 326-335 (1997).

6. N. Mohandas, Y. R. Kim, D. H. Tycko, J. Orlik, J. Wyatt, and W. Groner, "Accurate and independent measurement of volume and hemoglobin concentration of individual red cells by laser light scattering," Blood 68, 506-513 (1986).

7. J. R. Mourant, J. P. Freyer, A. H. Hielscher, A. A. Eick, D. Shen, and T. M. Johnson, "Mechanisms of light scattering from biological cells relevant to noninvasive optical-tissue diagnostics," Appl. Opt. 37, 3586-3593 (1998).

8. A. H. Hielscher, J. R. Mourant, and I. J. Bigio, "Influence of particle size and concentration on the diffuse backscattering of polarized light from tissue phantoms and biological cell suspensions," Appl. Opt. 36, 125-135 (1997).

9. F. Bevilacqua, P. Marquet, O. Coquoz, and C. Depeursinge, "Role of tissue structure in photon migration through breast tissues," Appl. Opt. 36, 44-51 (1997).

10. I. S. Saidi, S. L. Jacques, and F. K. Tittel, "Mie and Rayleigh modeling of visible-light scattering in neonatal skin," Appl. Opt. 34, 7410-7418 (1995).

11. T. J. Farrell, M. S. Patterson, and B. Wilson, "A diffusion theory model of spatially resolved, steady-state diffuse reflectance for noninvasive determination of tissue optical properties in vivo," Med. Phys. 19, 879-888 (1992).

12. R. Doornbos, R. Lang, M. Aalders, F. Cross, and H. J. C. M. Sterenborg, "The determination of in vivo human tissue optical properties and absolute chromophore concentrations using spatially-resolved steady-state diffuse reflectance spectroscopy," Phys. Med. Biol. 44, 967-981 (1999).

13. E. L. Hull, M. G. Nichols, and T. H. Foster, "Quantitative broadband near-infrared spectroscopy of tissue-simulating phantoms containing erythrocytes," Phys. Med. Biol. 43, 33813404 (1998).

14. F. Bevilacqua, D. Piguet, P. Marquet, J. D. Gross, B. J. Tromberg, and C. Depeursinge, "In vivo local determination of tissue optical properties: applications to human brain," Appl. Opt. 38, 4939-4950 (1999).

15. R. Bays, G. Wagnières, D. Robert, D. Braichotte, J. F. Savary, P. Monnier, and H. van den Bergh, "Clinical determination of tissue optical properties by endoscopic spatially resolved reflectometry," Appl. Opt. 35, 1756-1766 (1996).

16. S. L. Jacques, A. Gutsche, J. Schwartz, L. Wang, and F. Tittel, "Video reflectometry to specify optical properties of tissue in vivo," in Medical Optical Tomography: Functional Imaging and Monitoring, G. J. Müller, B. Chance, R. R. Alfano, S. R. Arridge, J. Beuthan, E. Gratton, M. Kaschke, B. R. Masters, S. Svanberg, and P. van der Zee, eds., Vol. ISII of SPIE Institute Series (Society for Photo-Optical Instrumentation Engineers, Bellingham, Wash., 1993), pp. 211-226.

17. A. Kienle, L. Lilge, M. S. Patterson, R. Hibst, R. Steiner, and B. C. Wilson, "Spatially resolved absolute diffuse reflectance measurements for noninvasive determination of the optical scattering and absorption coefficients of biological tissue," Appl. Opt. 35, 2304-2314 (1996).

18. L. Wang and S. L. Jacques, "Use of a laser beam with an oblique angle of incidence to measure the reduced scattering 
coefficient of a turbid medium," Appl. Opt. 34, 2362-2366 (1995).

19. R. Splinter, G. A. Nanney, L. Littman, C. H. Chuang, R. H. Svenson, J. R. Tuntelder, and G. P. Tatsis, "Monitoring tissue optical characteristics in situ using a CCD camera," Laser Life Sci. 6, 15-25 (1994).

20. F. Bevilacqua and C. Depeursinge, "Monte Carlo study of diffuse reflectance at source-detector separations close to one transport mean free path,” J. Opt. Soc. Am. A 16, 2935-2945 (1999).

21. F. Bevilacqua, "Local optical characterization of biological tissues in vitro and in vivo," Ph.D. dissertation (Swiss Federal Institute of Technology, Lausanne, Lausanne, Switzerland, 1998).

22. V. Venugopalan, J. S. You, and B. J. Tromberg, "Radiative transport in the diffusion approximation: an extension for highly absorbing media and small source-detector separations," Phys. Rev. E 58, 2395-2407 (1998).

23. A. Kienle and M. S. Patterson, "Determination of the optical properties of semi-infinite turbid media from frequencydomain reflectance close to the source," Phys. Med. Biol. 42, 1801-1819 (1997)

24. L. O. Svaasand, B. J. Tromberg, P. Wyss, M.-T. WyssDesserich, Y. Tadir, and M. W. Berns, "Light and drug distribution with topically administered photosensitizers," Lasers Med. Sci. 11, 261-265 (1996).

25. L. O. Svaasand, L. T. Norvang, E. J. Fiskerstrand, E. K. S. Stopps, M. W. Berns, and J. S. Nelson, "Tissue parameters determining the visual appearance of normal skin and portwine stains," Lasers Med. Sci. 10, 55-65 (1995).

26. M. G. Nichols, E. L. Hull, and T. H. Foster, "Design and testing of a white-light, steady-state diffuse reflectance spectrometer for determination of optical properties of highly scattering systems," Appl. Opt. 36, 93-104 (1997).

27. P. Marquet, F. Bevilacqua, C. Depeursinge, and E. B. de Haller, "Determination of reduced scattering and absorption coefficients by a single charge-coupled-device array measurement. I. Comparison between experiments and simulations," Opt. Eng. 34, 2055-2063 (1995).

28. D. M. Haaland, H. D. T. Jones, and E. V. Thomas, "Multivariate classification of the infrared spectra of cell and tissue samples,” Appl. Spectrosc. 51, 340-345 (1997).
29. H. Key, R. E. Davies, P. C. Jackson, and P. N. Wells, "Optical attenuation characteristics of breast tissues at visible and near-infrared wavelengths," Phys. Med. Biol. 36, 579-590 (1991).

30. V. G. Peters, D. R. Wyman, M. S. Patterson, and G. L. Frank, "Optical properties of normal and diseased human breast tissues in the visible and near infrared," Phys. Med. Biol. 35, 1317-1334 (1990).

31. W. H. Steel, Interferometry (Cambridge University Press, Cambridge, 1983).

32. F. A. Duck, Physical Properties of Tissue (Academic, London, 1990).

33. H. J. van Staveren, C. J. M. Moes, J. van Marle, S. A. Prahl, and M. J. C. van Gemert, "Light scattering in Intralipid-10\% in the wavelength range of 400-1100 nm," Appl. Opt. 30, 45074514 (1991).

34. D. R. Wyman, M. S. Patterson, and B. C. Wilson, "Similarity relations for anisotropic scattering in Monte Carlo simulations of deeply penetrating neutral particles," J. Comput. Phys. 81, 137-150 (2000).

35. C. de Boor, A Practical Guide to Splines (Springer-Verlag, New York, 1978).

36. The MathWorks, Inc., MATLAB Reference Guide (MathWorks, Natick, Mass., 1994).

37. C. L. Lawson and R. J. Hanson, Solving Least Squares Problems (Prentice-Hall, New York, 1974).

38. J. S. Dam, P. E. Andersen, T. Dalgaard, and P. E. Fabricius, "Determination of tissue optical properties from diffuse reflectance profiles by multivariate calibration,” Appl. Opt. 37, 772778 (1998).

39. A. Kienle and M. S. Patterson, "Improved solutions of the steady-state and the time-resolved diffusion equations for reflectance from a semi-infinite turbid medium," J. Opt. Soc. Am. A 14, 246-254 (1997).

40. J. R. Mourant, J. Boyer, A. H. Hielscher, and I. J. Bigio, "Influence of scattering phase function on light transport measurements in turbid media performed with small sourcedetector separations," Opt. Lett. 21, 546-548 (1996).

41. J. W. Pickering, S. A. Prahl, N. van Wieringen, J. F. Beek, H. J. C. M. Sterenborg, and M. J. C. van Gemert, "Doubleintegrating-sphere system for measuring the optical properties of tissue," Appl. Opt. 32, 399-410 (1993). 